\title{
Safety and Pharmacokinetics of Intravesical Chitosan/Interleukin-12 Immunotherapy in Murine Bladders
}

\author{
Khue G. Nguyen ${ }^{\mathrm{a}}$, Ethan S. Wagner ${ }^{\mathrm{a}}$, Maura R. Vrabel ${ }^{\mathrm{a}}$, Siena M. Mantooth ${ }^{\mathrm{a}}$, \\ Danielle M. Meritet ${ }^{\mathrm{b}}$ and David A. Zaharoff $\mathrm{f}^{\mathrm{a}, *}$

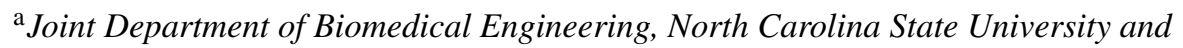 \\ University of North Carolina-Chapel Hill, Raleigh, NC, USA \\ ${ }^{\mathrm{b}}$ Department of Population Health and Pathobiology, College of Veterinary Medicine, \\ North Carolina State University, Raleigh, NC, USA
}

Received 20 April 2021

Accepted 30 September 2021

Pre-press 25 October 2021

Published 13 December 2021

\begin{abstract}
.
BACKGROUND: Intravesical administration of interleukin 12 (IL-12) co-formulated with the biopolymer, chitosan (CS/IL12), has demonstrated remarkable antitumor activity against preclinical models of bladder cancer. However, given historical concerns regarding severe toxicities associated with systemic IL-12 administration in clinical trials, it is important to evaluate the safety of intravesical CS/IL-12 prior to clinical translation.

OBJECTIVE: To evaluate the pharmacokinetics as well as the local and systemic toxicities of intravesical CS/IL-12 immunotherapy in laboratory mice.

METHODS: Local inflammatory responses in mouse bladders treated with intravesical IL-12 or CS/IL-12 were assessed via histopathology. Serum cytokine levels following intravesical and subcutaneous (s.c.) administrations of IL-12 or CS/IL-12 in laboratory mice were compared. Systemic toxicities were evaluated via body weight and liver enzyme levels.

RESULTS: Intravesical IL-12 and CS/IL-12 treatments did not induce significant local or systemic toxicity. IL-12 dissemination and exposure from intravesical administration was significantly lower compared to s.c. injections. Weekly intravesical CS/IL-12 treatments were well-tolerated and did not result in blunted immune responses.

CONCLUSIONS: Intravesical CS/IL-12 is safe and well-tolerated in mice. In particular, the lack of cystitis and acute inflammation justifies continued investigation of intravesical CS/IL-12 immunotherapy in larger animals and patients with bladder cancer.
\end{abstract}

Keywords: Bladder cancer, Interleukin-12, immunotherapy, chitosan, intravesical

${ }^{*}$ Correspondence to: David A. Zaharoff, Ph.D., Joint Department of Biomedical Engineering, North Carolina State University \& University of North Carolina-Chapel Hill, 4130 Engineering Building III, Campus Box 7115, Raleigh, NC 27695, USA. Tel.: +19195156757; E-mail: dazaharo@ncsu.edu.

\section{INTRODUCTION}

The vast majority of bladder cancers are diagnosed relatively early with non-muscle invasive disease found in $70-80 \%$ of patients at presentation. 
Unfortunately, bladder cancer has one of the highest recurrence rates of any cancer - up to 65\% [1]. Much of the high recurrence rate is driven by highgrade non-muscle invasive bladder cancer (NMIBC) which has a strong tendency to invade the detrusor and spread to other organs. Since the pioneering work of Morales et al in 1976 [2], Mycobacterium bovis Bacillus Calmette-Guerin (BCG) has been the standard-of-care intravesical therapy for high-grade NMIBC. BCG induces a local inflammatory response which includes the production of immunostimulatory cytokines and chemokines (reviewed in [3]). This inflammatory response induces a large influx of granulocytes, macrophages and lymphocytes into the bladder wall [4-6]. Unfortunately, about 20-30\% of patients fail initial BCG therapy and 30-50\% of BCG responders develop recurrences within 5 years $[7,8]$. Additional therapies are needed to limit progressive recurrences and to improve overall survival for both BCG responders and nonresponders.

IL-12 is a $\mathrm{T}_{\mathrm{H}}$ 1-polarizing cytokine that has demonstrated remarkable antitumor activity against a range of malignancies in preclinical studies [9-11]. However, severe toxicities associated with systemic injections of IL-12 have limited its clinical use as an anticancer agent [12]. A recent renaissance of IL-12-based immunotherapies have capitalized on innovative strategies to localize IL-12 to the tumor microenvironment thus limiting toxic systemic exposure [11]. Intravesical delivery is one such localized approach that seeks to maximize the exposure of an anticancer agent to a bladder tumor while minimizing systemic distribution [13, 14].

For more than ten years, we have explored intravesical administration of IL-12 co-formulated with the cationic biopolymer, chitosan (CS), to treat orthotopic bladder tumors in mice [15-17]. CS is a versatile, abundant, and natural copolymer of glucosamine and $\mathrm{N}$-acetylglucosamine that has been used as a pharmaceutical excipient, a weight loss supplement and as an active component of FDAapproved hemostatic dressings [18, 19]. Our interest in CS as an intravesical delivery enhancer stems from its ability to coat mucosal surfaces while loosening gap junctions potentially allowing better penetration of drugs into the bladder wall [16, 17, 20]. In 2009, we first demonstrated that intravesical chitosan/IL12 could eliminate lethal orthotopic bladder tumors in mice [15]. Furthermore, with an $88-100 \%$ cure rate, intravesical CS/IL-12 far outperformed intravesical BCG (0\% cure rate). CS/IL-12 treatment was associated with robust $\mathrm{T}$ cell infiltration of bladder tumors and all mice that were cured following intravesical CS/IL-12 were completely protected from a live orthotopic tumor rechallenge. Subsequent studies demonstrated that intravesical CS/IL-12 induced protective immunity against distant (s.c.) tumor challenges as well [16]. This publication was also the first to demonstrate that the majority of abscopal s.c. bladder tumors, mimicking metastatic lesions, could be eliminated following treatment of orthotopic bladder tumors with intravesical CS/IL-12. Interestingly, when mice with naïve bladders were treated with intravesical CS/IL-12, s.c. tumors were delayed, but still progressed. Taken together, these findings indicated that an intravesical CS/IL-12 immunotherapy can induce systemic immunity. Additional experiments confirmed that cytotoxic $\mathrm{T}$ lymphocytes (CTLs) were tumor-specific and could lyse bladder cancer cells at high levels [16]. Additional mechanistic studies revealed that multiple administrations of CS/IL-12 are needed for optimal effect [17]. Early responses to the initial 1 or 2 instillations are dominated by an influx of innate immune cells such as inflammatory macrophages and granulocytes. Subsequent administrations led to a robust adaptive immune response led by $\mathrm{CD} 4^{+}$and $\mathrm{CD} 8^{+}$effector memory T cells. Depletion studies demonstrated that $\mathrm{CD}^{+}{ }^{+} \mathrm{T}$ cells and to a lesser extent $\mathrm{CD} 4^{+} \mathrm{T}$ cells were required for tumor elimination and long-term survival [17].

In anticipation of clinical translation, we have narrowed our focus to assess the safety of intravesical CS/IL-12. In the past decade, we have treated and eliminated established murine orthotopic MB49, MB49.luc and MBT-2 tumors in many mice. Although we have never witnessed obvious clinical signs of toxicity, the local and systemic immunotoxicology of intravesical CS/IL-12 has not been investigated thoroughly. The current studies fill this gap by evaluating the safety and pharmacokinetics of intravesically administered CS/IL-12. Specifically, serum cytokine levels in mice following treatment were measured to assess pharmacokinetics. Dose escalation studies were performed to determine the effect of dose on peak cytokine levels. Elevated liver enzymes, a common adverse event associated with systemic IL-12 exposure [12, 21] were investigated via alanine aminotransferase (ALT) activity measurements. Potential local inflammatory effects on bladder tissues were evaluated via histopathological analyses. Importantly, different cohorts of naïve or tumor-bearing mice were treated with unformulated 
IL-12 or CS/IL-12 delivered via subcutaneous or intravesical routes. This experimental design allowed for direct comparisons of formulations, tumor status and routes of administration.

\section{MATERIALS AND METHODS}

\section{Animals}

Experiments were performed in 8 to 12 -week-old female C57BL/6 mice (Charles River Laboratories, Wilmington, MA). Only female mice were used due to technical challenges associated with catheterization of male mice. Animal use was in compliance with the Public Health Service Policy on Human Care and Use of Laboratory Animals. All experiments involving laboratory animals were approved by the Institutional Animal Care and Use Committee at North Carolina State University (Protocol \#18-045).

\section{Recombinant IL-12 and chitosan}

Recombinant mouse IL-12 (mIL-12) was produced and purified in-house as described previously [22-24]. Briefly, mIL-12 was overexpressed by HEK293 cells stably transfected with a plasmid encoding mIL-12. IL-12-producing HEK293 cells were grown in serum-free media in a hollow fiber bioreactor (FiberCell Systems Inc., New Market, MD) connected to a Duet Pump (FiberCell Systems Inc., New Market, MD). mIL-12 was purified from supernatants via heparin sepharose chromatography as described previously [22-24]. Chitosan acetate (30 - $200 \mathrm{kDa}, 80-90 \%$ deacetylated) was obtained from Heppe Medical Chitosan GmbH (Germany). All CS/IL-12 formulations consisted of mIL-12 added to a $1 \%(w / v)$ chitosan acetate solution in Dulbecco's phosphate-buffered saline (DPBS).

\section{Intravesical and subcutaneous treatments}

IL-12 or CS/IL-12 was administered via either intravesical instillations or s.c. injections. For intravesical treatment, mice were anesthetized with i.p. ketamine $(15 \mathrm{mg} / \mathrm{kg}) / x y l a z i n e(75 \mathrm{mg} / \mathrm{kg})$. A $24 \mathrm{G} \times$ 3/4-inch Teflon catheter (Terumo) was then inserted into the bladder through the urethra. One hundred microliters of IL-12 or CS/IL-12 were then administered intravesically for 30 minutes while catheters/ syringes were kept in place. Subcutaneous treatments consisted of $50 \mu \mathrm{L}$ of IL-12 or CS/IL-12 injected into the shaved flanks of isoflurane-anesthetized mice.

\section{Histopathology}

Cohorts of mice treated with DPBS, CS alone, IL12 alone or CS/IL-12 were sacrificed $24,48,72$ or $96 \mathrm{hrs}$ after treatment. Urinary bladders $(n=2$ per treatment/per timepoint; $n=1$ for DPBS) were collected, fixed (10\% neutral buffered formalin), paraffin embedded and sectioned at $5 \mu \mathrm{m}$ thickness for histology slide preparation. For each treatment group, two transverse sections from two urinary bladders were represented on a single slide. Hematoxylin and eosinstained slides were blindly viewed using Aperio eSlide Manager (Leica Biosystems, Buffalo Grove, IL) and Aperio ImageScope 12.4 (Leica Biosystems, Buffalo Grove, IL) by a board-certified veterinary anatomic pathologist (DM). A four-step grading system was devised for inflammation severity based on current recommended toxicologic pathology standards [25].

\section{Cytokine and hepatotoxicity measurements}

Blood was collected via mandibular/facial vein bleeding at specified time points after treatment. No more than $1 \%$ of an animal's body weight was collected over any $48 \mathrm{hr}$ period. Blood was allowed to clot for $1 \mathrm{hr}$ at room temperature before centrifugation at $1,500 \times g$ for 15 minutes to collect sera. Serum levels of IL-12 and IFN- $\gamma$ were measured via enzymelinked immunosorbent assay (ELISA) (88-7121-88 (IL-12); 88-7314-88 (IFN- $\gamma$ ); Thermo Fisher). Area under-the-curve (AUC) was calculated by application of the trapezoidal method using GraphPad Prism 9. Serum ALT activity levels were determined via an ALT activity assay kit (ab105134; Abcam). Mice treated with four consecutive daily i.p. injections of unformulated IL-12 served as a positive control for ALT activity.

\section{Tumor implantation and treatment}

Orthotopic bladder tumors were established in female C57BL/6 mice as described previously [15]. Briefly, mice were anesthetized with ketamine $(15 \mathrm{mg} / \mathrm{kg}) / x y l a z i n e(75 \mathrm{mg} / \mathrm{kg})$ prior to inserting a 24 G x 3/4" IV catheter (Terumo, Somerset, NJ) into the bladder and delivering $100 \mu \mathrm{L}$ of poly-L-lysine solution (PLL, MW =70,000 to 150,000) for $10 \mathrm{~min}$, followed by $100 \mu \mathrm{L}$ containing 75,000 MB49 cells 


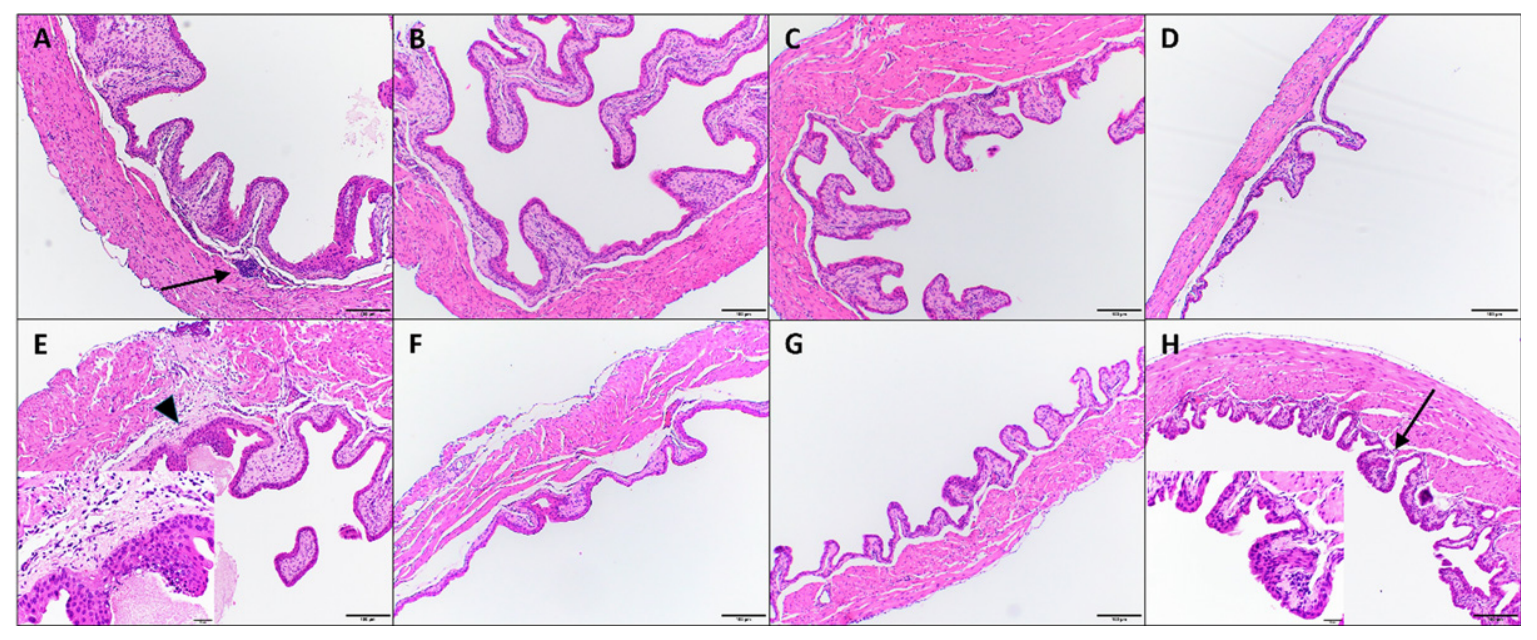

Fig. 1. Histologic images of mouse urinary bladders at different time points. Images A-D. IL-12 alone treatment at $24 \mathrm{~h}$ (A), 48 hours (B), 72 hours (C) and 96 hours (D). Images E-H. CS/IL-12 treatment at 24h (E), 48 hours (F), 72 hours (G) and 96 hours (H). Arrows indicate minimal lymphoplasmacytic perivascular infiltrates that constituted a grade 1 response; insert for image $(\mathrm{H})$ shows inflammation at higher magnification. Arrowhead indicates rare, minimal neutrophilic inflammation and fibrin exudation (also grade 1); insert for image (E) shows inflammation at higher magnification. All images using 10x objective. Inserts for $(\mathrm{E})$ and $(\mathrm{H})$ are using 40x objective.

Table 1

Urinary bladder inflammation severity** with treatment and time after treatment

\begin{tabular}{|c|c|c|c|c|c|}
\hline & \multicolumn{4}{|c|}{ Time after treatment } & \multirow[t]{2}{*}{ Type(s) of inflammation } \\
\hline & $24 \mathrm{hrs}$ & $48 \mathrm{hrs}$ & $72 \mathrm{hrs}$ & $96 \mathrm{hrs}$ & \\
\hline DPBS & 1 & 1 & 1 & $\mathrm{n} / \mathrm{a}$ & Lymphoplasmacytic, perivascular \\
\hline $\mathrm{CS}$ & $0 / 0$ & $1^{*}$ & $1^{*}$ & $1 / 0$ & $\begin{array}{l}\text { Lymphoplasmacytic, perivascular; one instance of neutrophilic } \\
\text { infiltrate with submucosal edema }\end{array}$ \\
\hline IL-12 & $1^{*}$ & $1 / 0$ & $1 / 0$ & $1 / 1$ & Lymphoplasmacytic, perivascular \\
\hline CS/IL-12 & $1 / 1$ & $0 / 0$ & $1 / 0$ & $1 / 1$ & $\begin{array}{l}\text { Lymphoplasmacytic, perivascular; one instance of neutrophilic } \\
\text { infiltrate with fibrin exudation }\end{array}$ \\
\hline
\end{tabular}

*only 1 grade was given when individual bladders on a slide could not be distinguished. ${ }^{* *} 0=$ no inflammation; $1=$ minimal inflammation; $2=$ mild inflammation; 3 = moderate inflammation; $4=$ marked inflammation.

for 30 to $45 \mathrm{~min}$. Mice were monitored daily for development of hematuria. Ten days after implantation, mice with frank hematuria and a cohort of naïve mice, received intravesical immunotherapy with CS/IL-12. Blood was collected $6 \mathrm{hrs}$ and $48 \mathrm{hrs}$ after treatment to measure serum IL-12, IFN $\gamma$ and ALT levels as described above.

\section{Statistical analysis}

Differences in serum cytokine levels and ALT levels were compared via two-way ANOVA with Sidak's posttest. Comparisons of bodyweight measurements were performed via two-way ANOVA with Dunnett's posttest to compare individual treatment groups versus the control group (PBS treated mice). Statistical significance was accepted at the $p \leq 0.05$ level. All analyses were conducted using GraphPad Prism 9 software (GraphPad Software, CA).

\section{RESULTS}

\section{Histopathological analyses reveal only minor inflammation of bladder tissues following intravesical treatments}

Urinary bladders from mice receiving intravesical CS alone, IL-12 alone or CS/IL-12 were harvested up to $96 \mathrm{hrs}$ after treatments for histopathological analyses (Fig. 1). Inflammation severity grade by slide/treatment group are shown in Table 1. Where inflammation severity varied, a grade was assigned according to areas of more severe inflammation. Distribution and tissue injury - such as ulceration, edema and fibrin exudation - were noted in Table 1. Tissue injury interpreted as a result of test article administration (i.e., focal hemorrhage with or without erythrophagocytes present) was not considered when assigning a grade. None of the intravesical treatments 

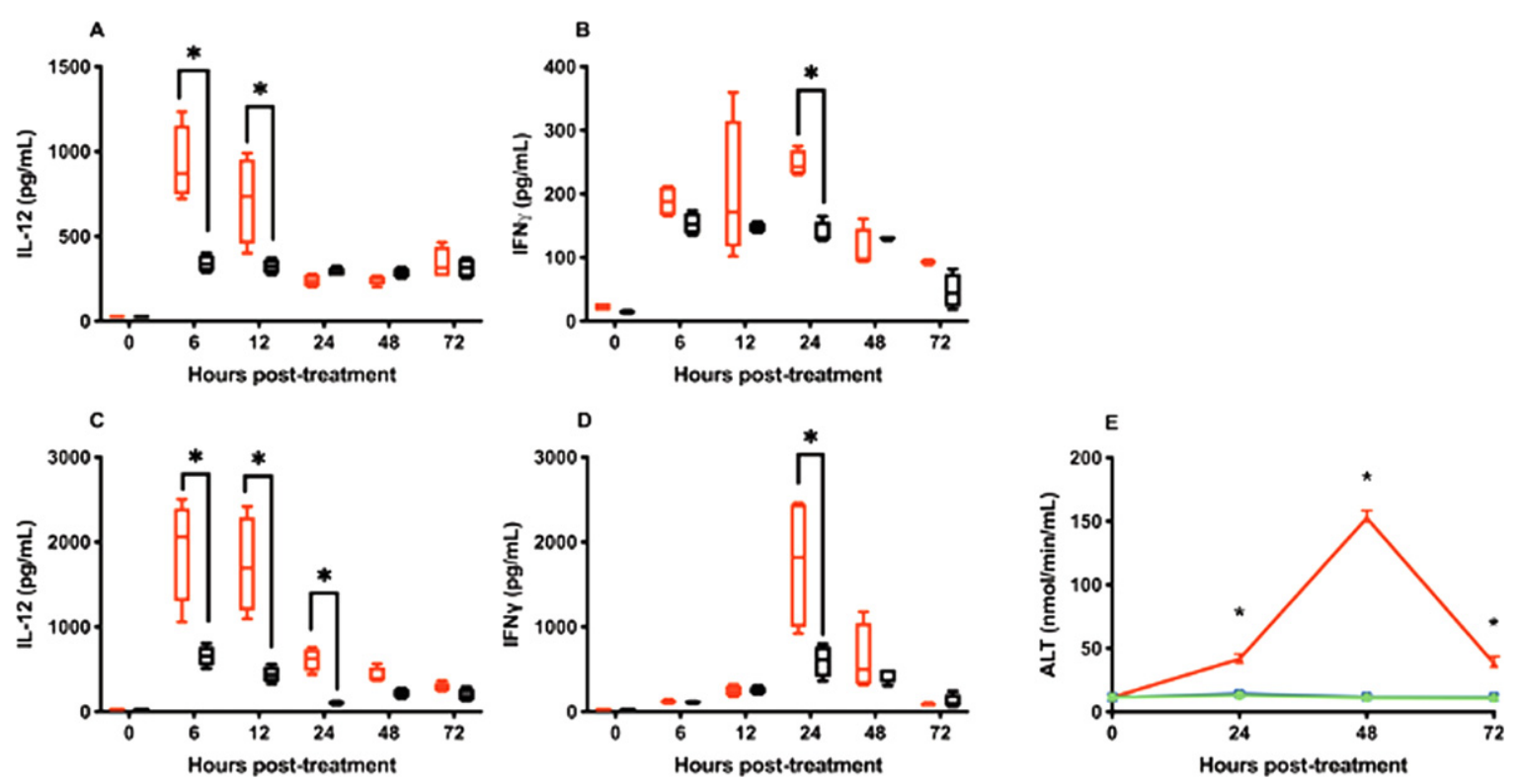

Fig. 2. Chitosan in combination with intravesical instillations retain IL-12 releases. Mice were treated intravesically or subcutaneously with IL-12 (red bars) or CS/IL-12 (black bars). Sera were obtained using facial vein bleeding at multiple time points $(0 \mathrm{~h}, 6 \mathrm{~h}, 12 \mathrm{~h}, 24 \mathrm{~h}$, $48 \mathrm{~h}$, or $72 \mathrm{~h}$ ). The serum releases of IL-12 (A, C) or IFN- $\gamma(\mathrm{B}, \mathrm{D})$ in responses to intravesical treatments of IL-12 and CS/IL-12 (A, B), or subcutaneous treatments of IL-12 and CS/IL-12 (C, D) were measured via ELISA. Asterisks indicate a significant difference of IL-12 or IFN- $\gamma$ levels in responses to IL-12 or CS/IL-12 treatments $\left(^{*}, p<0.05\right.$ via two-way ANOVA with Sidak's posttest). Hepatotoxicity (E) was determined by measuring the activity of alanine transaminase released in mouse sera in response to intravesical administrations of IL-12 (blue squares) or CS/IL-12 (green circles), and 4 consecutive daily doses of i.p. IL-12 (red triangles). Asterisks indicate a significant difference of ALT activity in responses to IL-12, CS/IL-12 or $4 \times$ i.p. IL-12 treatments $\left(^{*}, p<0.05\right.$ via two-way ANOVA with Sidak's posttest). Experiments were performed in triplicate and repeated with similar results.

resulted in inflammation severity above grade 1 out of 4 . There was also no noticeable effect of timing with respect to treatment.

\section{Pharmacokinetics of IL-12 and CS/IL-12}

treatments show limited systemic exposure

following intravesical administration

Serum IL-12 levels following intravesical administration peaked at $924.19 \pm 192.23 \mathrm{pg} / \mathrm{ml}$ and $340.45 \pm 42.55 \mathrm{pg} / \mathrm{ml}$ at $6 \mathrm{hrs}$ after treatment for IL12 and CS/IL-12 treatments, respectively (Fig. 2A). Peak serum IL-12 levels following s.c. injections were roughly twice that of the intravesical route at $1921.39 \pm 531.16 \mathrm{pg} / \mathrm{ml}$ and $654.20 \pm 104.21 \mathrm{pg} / \mathrm{ml}$, respectively, at $6 \mathrm{hrs}$ after treatment (Fig. 2C). With respect to total exposure, $\mathrm{AUC}_{\mathrm{IL}-12}$ for IL12 and CS/IL-12 were $2306 \pm 248.5(\mathrm{pg} / \mathrm{mL}) \times$ hour and $1410 \pm 60.15(\mathrm{pg} / \mathrm{mL}) \times$ hour for intravesical administration and $4842 \pm 604.0(\mathrm{pg} / \mathrm{mL}) \times$ hour and $1529 \pm 120.2(\mathrm{pg} / \mathrm{mL}) \times$ hour for s.c. injections.

IFN- $\gamma$, a $T_{H} 1$ cytokine produced by lymphocytes in response to IL-12, is a key mediator of IL-12's toxicity [26-28]. As expected IFN- $\gamma$ reached peak levels about 24 hours after IL-12 delivery for both intravesical and s.c. routes (Fig. 2B and D). Similar to IL-12, far less IFN- $\gamma$ was found in circulation following intravesical administration compared to s.c. administration (Fig. 2B and D).

One of the primary toxicities associated with IL-12 administration is hepatotoxicity mediated by IFN- $\gamma$ $[29,30]$. To determine if the above serum IFN- $\gamma$ levels induced by intravesical IL-12 or CS/IL-12 were able to induce hepatotoxicity, ALT activity levels were quantified 24,48 and $72 \mathrm{hrs}$ after intravesical treatments. Neither intravesical IL-12 nor CS/IL-12 caused ALT levels to rise above pre-treatment averages (Fig. 2E). In contrast, 4 consecutive daily i.p. injections of IL-12, a regimen known to induce hepatotoxicity [12], generated about a 13-fold increase in ALT that peaked at $48 \mathrm{hrs}$ after the last injection (Fig. 2E).

\section{Multiple intravesical IL-12 or CS/IL-12 treatments have minimal impact on IFN- $\gamma$ production}

Repeated systemic IL-12 treatments can result in blunted immune responses. This is most evident by 

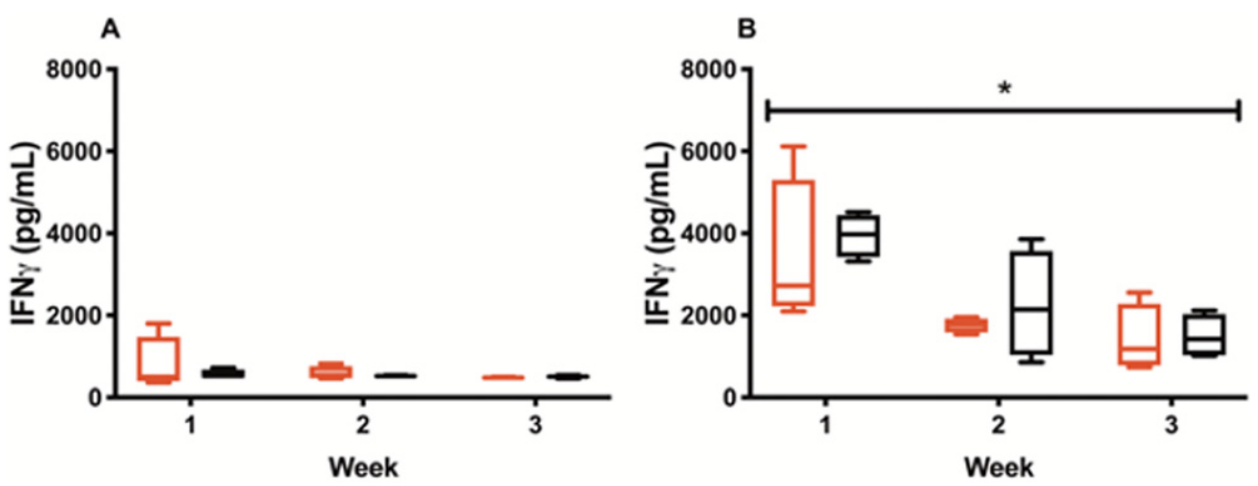

Fig. 3. Declining of IFN- $\gamma$ triggered by repeated IL-12 treatments. Mice were treated intravesically or subcutaneously with IL-12 (red bars) or CS/IL-12 (black bars) in 3 consecutive weeks. Sera were obtained using facial vein bleeding at $24 \mathrm{~h}$ after administrations every week. The serum releases of IFN- $\gamma$ in responses to intravesical treatments of IL-12 and CS/IL-12 (A) or subcutaneous treatments of IL-12 and CS/IL-12 (B) were measured via ELISA. Asterisks indicate a significant difference of IFN- $\gamma$ levels in responses to IL-12 or CS/IL-12 treatments in the period of 3 weeks $\left({ }^{*}, p<0.05\right.$ via two-way ANOVA). Experiments were performed in triplicate and repeated with similar results.

a reduction in IFN- $\gamma$ production $[31,32]$. To determine how repeated intravesical instillations of IL-12 or CS/IL-12 affect IFN- $\gamma$ production in mice, serum IFN- $\gamma$ levels at $24 \mathrm{hrs}$ after each of 3 weekly treatments were examined.

For the s.c. injections, IFN- $\gamma$ levels dropped from $3416.49 \pm 1582.46 \mathrm{pg} / \mathrm{mL}$ following the first IL12 alone injection (week 1) to $1761.40 \pm 151.87 \mathrm{pg}$ / $\mathrm{mL}$ (week 2) and $1416.57 \pm 709.66 \mathrm{pg} / \mathrm{mL}$ (week 3) (Fig. 3B). Similar results were observed for CS/ IL-12 s.c. injections decreasing from $3946.60 \pm$ $457.96 \mathrm{pg} / \mathrm{mL}$ (week 1) to $2251.43 \pm 1139.51 \mathrm{pg} / \mathrm{mL}$ (week 2) and $1496.54 \pm 466.04 \mathrm{pg} / \mathrm{mL}$ (week 3) (Fig. 3B). The decrease in IFN- $\gamma$ levels with multiple injections was statistically significant $(p<0.05$ vs two-way ANOVA).

For intravesical treatments, IFN- $\gamma$ levels were $790.59 \pm 586.11 \mathrm{pg} / \mathrm{mL}$ following the first injection (week 1) to $595.47 \pm 139.46 \mathrm{pg} / \mathrm{mL}$ (week 2) to $485.46 \pm 8.54$ (week 3 ) for IL-12 alone and $565.43 \pm$ $94.43 \mathrm{pg} / \mathrm{mL}$ (week 1), to $525.15 \pm 8.61 \mathrm{pg} / \mathrm{mL}$ (week 2) to $505.46 \pm 29.73 \mathrm{pg} / \mathrm{mL}$ (week 3 ) for CS/ IL-12 (Fig. 3A). Although IFN- $\gamma$ levels trended downward, there was no statistical differences between week 1, 2 and 3 measurements for intravesical CS/IL-12 immunotherapy ( $p>0.05$ vs two-way ANOVA).

\section{Escalating doses of intravesical CS/IL-12 are well-tolerated}

Although we previously demonstrated that $1 \mu \mathrm{g}$ of IL-12 formulated with CS is effective at clearing nearly all orthotopic bladder tumors [15-17], it was important to explore the safety of higher doses which may be required to manage bladder tumors in other species, including dogs and humans. Thus, a dose escalation study of IL-12 or CS/IL-12 at 1,5 , and $10 \mu \mathrm{g} / \mathrm{mouse}$ was performed. Surprisingly, increasing the IL-12 dose by 5 or 10 times did not significantly increase the amount of IFN- $\gamma$ found in serum following intravesical administration of IL-12 or CS/IL-12 ( $p>0.05$ via ANOVA). As in previous experiments, formulation with CS did not influence systemic IFN- $\gamma$ levels following intravesical treatments ( $p>0.05$ IL-12 vs CS/IL-12) (Fig. 4A). For subcutaneous injections, IFN- $\gamma$ production increased at higher IL-12 doses following IL-12 alone treatments, but not CS/IL-12 treatments (Fig. 4B). Similar to previous experiments (Fig. 2), CS formulation reduced systemic IFN- $\gamma$ levels compared to IL-12 alone injections ( $p<0.05$ via ANOVA).

None of the mice receiving any of the IL-12 or CS/IL-12 treatments exhibited any signs of distress. As a more objective measure of toxicity, daily bodyweight measurements confirmed that intravesical immunotherapy with CS/IL-12 at 1,5 or $10 \mu \mathrm{g}$ IL-12 did not induce significant weight loss compared to PBS treated mice ( $p>0.05$ via two-way ANOVA) (Fig. 4C). S.c. injections of higher doses of CS/IL12 caused minor, transient weight loss that was fully restored by $96 \mathrm{hrs}$ after treatment (Fig. 4D).

\section{Bladder tumors do not influence IL-12 uptake}

Because bladder tumors are highly vascularized and bloody, as evidenced by the hematuria that so often accompanies a bladder cancer diagnosis, it was 

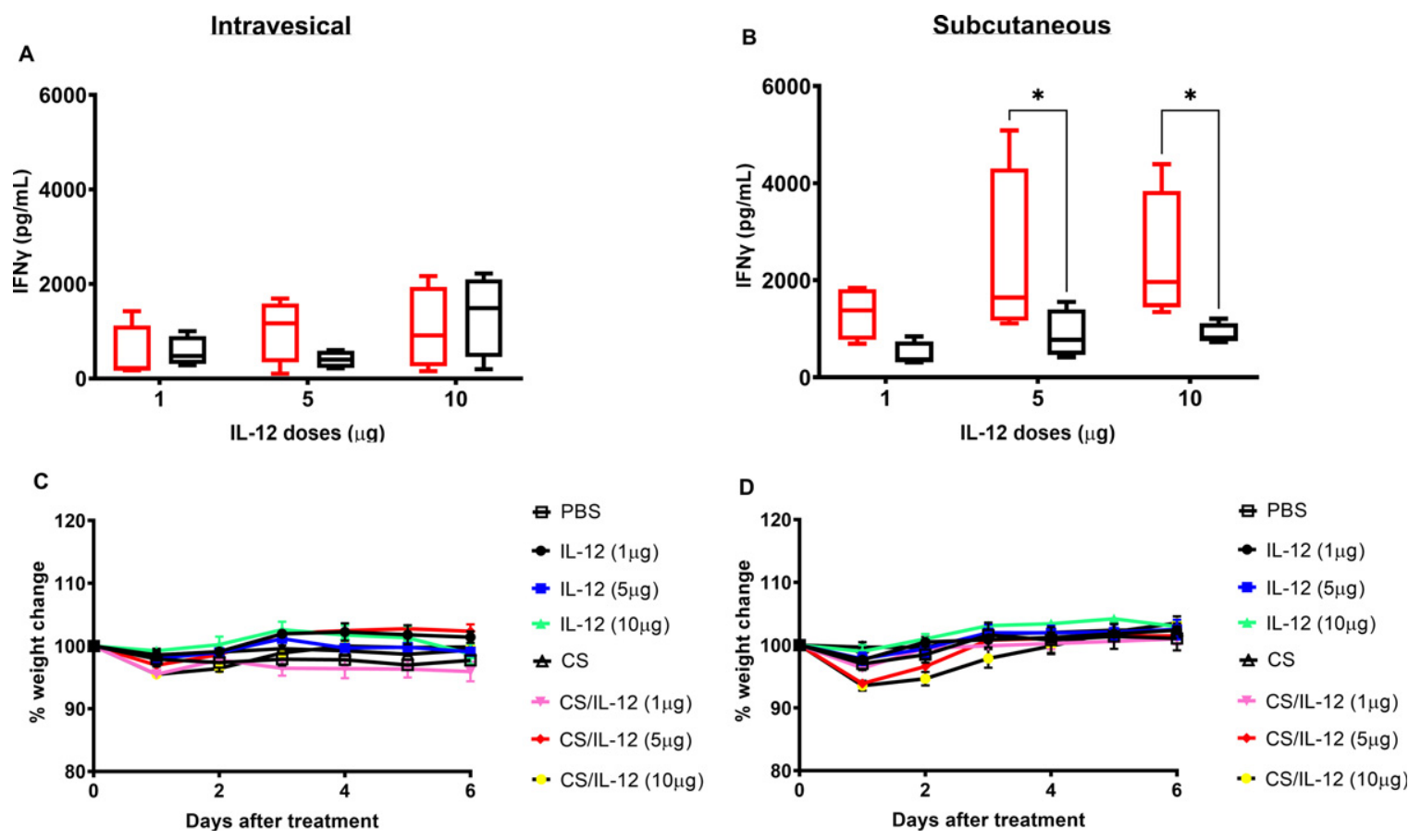

Fig. 4. Dose escalations of intravesical IL-12 and chitosan are well-tolerated. Mice were treated intravesically or subcutaneously by multiple doses of IL-12 ( $1 \mu \mathrm{g}$ IL-12/mouse, $5 \mu \mathrm{g}$ IL-12/mouse, or $10 \mu \mathrm{g}$ IL-12/mouse) without or with the presence of $1 \%$ CS (red bars or black bars, respectively). Sera were obtained using facial vein bleeding at $24 \mathrm{~h}$ after administrations. The serum releases of IFN- $\gamma$ in responses to intravesical or subcutaneous treatments of CS/IL-12 (A) and intravesical or subcutaneous treatments of IL-12 (B) were measured via ELISA. Asterisks indicate a significant difference of IFN $\gamma$ levels in responses to IL-12 or CS/IL-12 treatments $\left({ }^{*}, p<0.05\right.$ via two-way ANOVA). Mouse bodyweights in responding to intravesical treatments of IL-12 and CS/IL-12 (C) or subcutaneous treatments of IL-12 and CS/IL-12 (D) were monitored every day in a period of 6 days. Percent bodyweight changes were calculated based on the difference of bodyweight post-treatments and pre-treatments. Asterisks indicate a significant difference of bodyweight in responding to IL-12 or CS/IL-12 treatments $\left({ }^{*}, p<0.05\right.$ via two-way ANOVA with Dunnett's posttest). Experiments were performed in triplicate and repeated with similar results.

important to determine if the presence of a bladder tumor affected IL-12 absorption and associated toxicities following intravesical CS/IL-12 immunotherapy. For the $6 \mathrm{hr}$ measurement, which was chosen to correspond to the peak serum IL-12 level, there was no difference between naive and orthotopic MB49 bladder tumor-bearing mice $-420.0 \pm 191.1 \mathrm{pg} / \mathrm{ml}$ and $601.2 \pm 299.2 \mathrm{pg} / \mathrm{ml}$, respectively (Fig. 5A). At the $48 \mathrm{hr}$ timepoint, serum IFN $\gamma$ concentrations $(203.3 \pm 35.6 \mathrm{pg} / \mathrm{ml}$ and $156.4 \pm 6.3 \mathrm{pg} / \mathrm{ml})$ and ALT activity levels $(7.6 \pm 0.6 \mathrm{nmol} / \mathrm{min} / \mathrm{mL}$ and $7.1 \pm 0.6 \mathrm{nmol} / \mathrm{min} / \mathrm{mL}$ ) from naïve and tumorbearing mice were also statistically indistinguishable (Fig. 5B, C).

\section{DISCUSSION}

As mentioned above, we have not observed any signs of IL-12 treatment-related distress in the hundreds of bladder tumor-bearing mice that we have treated intravesically over the past $10+$ years. Nonetheless, our aspirations to translate CS/IL-12 benefit from a closer look at systemic IL-12 exposure and its potential downstream effects. While mice are not always reliable indicators of toxicity in humans, murine studies can provide important quantitative and qualitative data to guide clinical trial planning. Thus, the goals of these focused experiments were: 1) to assess systemic exposure to IL-12 following intravesical instillation; and 2) to determine the magnitude and scope of adverse events induced by intravesical CS/IL-12 immunotherapy. Subcutaneous injections of IL-12 and CS/IL-12 were used for reference as this is a well characterized systemic route which has resulted in serious adverse events in humans $[11,33]$.

Not surprisingly, systemic IL-12 exposure following intravesical CS/IL-12 was far lower than s.c. CS/IL-12 or IL-12 injections (Fig. 2). After a 30minute intravesical instillation, catheters are removed and most of the administered IL-12 is likely to be 
A

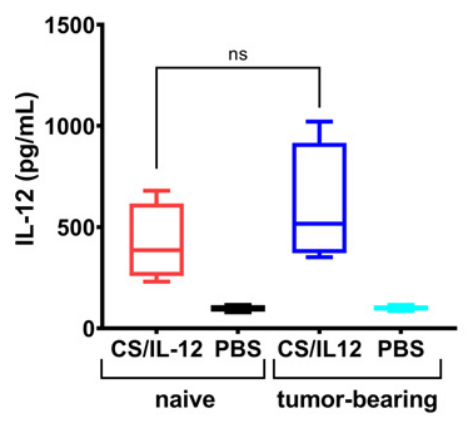

B

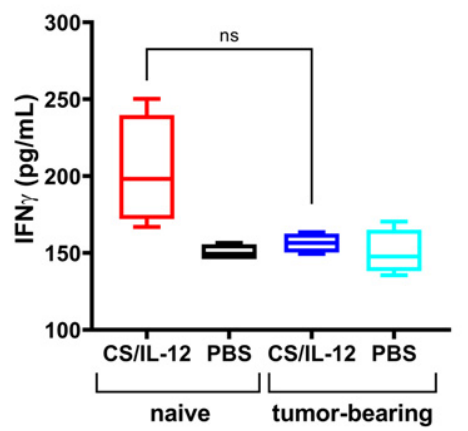

C

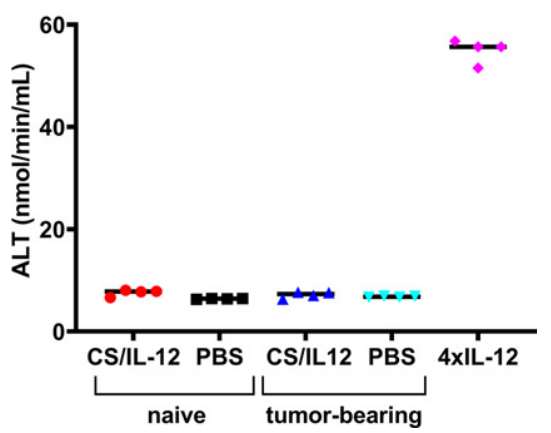

Fig. 5. Intravesical instillations of IL-12 were released at similar rate by chitosan in naïve mice and MB49 tumor bearing mice. Naïve mice were treated intravesically with CS/IL-12 (red bars) or PBS (black bars). MB49 tumor-bearing mice were treated intravesically with CS/IL-12 (blue bars) or PBS (cyan bars). Serum levels of IL-12 at 6 hrs after treatment (A) or IFN- $\gamma$ at 48 hrs after treatment (B) were measured via ELISA. Hepatotoxicity at $48 \mathrm{hrs}$ after treatment (C) was determined by measuring ALT activity in the sera of naive and MB49 tumor-bearing mice receiving intravesial CS/IL-12 or PBS. Naïve mice treated with four consecutive daily doses of i.p. IL-12 (1 $\mu \mathrm{g})$ served as a positive control of hepatotoxicity. "ns" indicates "not significant" ( $p>0.05$ via $t$-test).

excreted, whereas the full dose of IL-12 remains in the body following a s.c. injection. To get a sense of the maximum amount or dose of IL-12 that reaches the circulation following intravesical CS/IL-12 administration, we can multiply the peak concentration of $340.45 \pm 42.55 \mathrm{pg} / \mathrm{ml}$ by the total mouse blood volume of $1925-2000 \mu \mathrm{L}$ for a 25 gram mouse (77-80 $\mu \mathrm{L} /$ gram [34]) to find that about $680 \mathrm{pg}$ of IL-12 is in circulation. This is nearly a $1500-$ fold reduction from the initial $1 \mu \mathrm{g}$ CS/IL-12 dose. Similarly, unformulated IL-12 is effectively reduced by about 540 -fold. These data are encouraging from a safety perspective as the intravesical route minimizes systemic exposure to IL-12.

It was also encouraging to find that the presence of an orthotopic bladder tumor did not affect IL12 uptake and associated toxicities. Treatment of both naïve and tumor-bearing mice with intravesical CS/IL-12 resulted in similar levels of serum IL-12, IFN $\gamma$ and ALT (Fig. 5). This is notable as bladder tumors are well vascularized and leaky. These data should allow for direct translation into dogs and humans with bladder cancer and avoids unnecessary safety studies in healthy subjects.

Our data agree in principle with a recent publication evaluating the biodistribution of quantum dots (QDs) administered intravesically [14]. In fact, the authors found no statistically significant increases in cadmium, a component of QDs, in organs outside the bladder [14]. QDs are much larger than IL-12 and thus less systemic exposure can be expected. To our knowledge, the pharmacokinetics of chemotherapeutics, such as mitomycin $\mathrm{C}$, which are commonly used to treat NMIBC in humans, have not been described in murine models. However, one might expect that smaller drugs will be absorbed into the systemic circulation more readily than large, complex cytokines or nanoparticles. Nevertheless, in general, the intravesical route allows for maximal delivery of a therapeutic to bladder tissues with reduced systemic exposure.

Although measurable amounts of IL-12 and IFN$\gamma$ were found in circulation following intravesical IL-12 and CS/IL-12, this did not result in hepatotoxicity which is a typical adverse event associated with systemic IL-12 administration [12]. In fact, ALT levels were completely unaffected by intravesical immunotherapy either in the presence or absence of a tumor (Figs. 2E, 5C). Body weight measurements appeared to agree with the lack of systemic toxicity. Mice treated intravesically with the highest levels of CS/IL-12 experienced a transient decrease in weight, only about $4 \%$ on average. It should be noted that nearly all mice, including PBS treated controls, experienced some weight loss after the intravesical treatments which could be related to the ketamine/xylazine anesthesia [35]. Nevertheless, all mice quickly returned to pre-treatment body weights within a day or two. Not surprisingly, body weight changes following the s.c. injections with higher doses of CS/IL-12 were prolonged. This further supports the notion that there are considerable differences in toxicities between the two routes.

Our previous studies demonstrated that multiple treatments of CS/IL-12 were needed to completely eradicate bladder tumors in mouse models [16, 17]. However, numerous publications have shown that repeated IL-12 administration not only results in 
greater toxicity, but also a blunted IFN- $\gamma$ response due, in large part, to upregulation of immunosuppressive IL-10 [31, 32]. Indeed, our studies showed that weekly s.c. IL-12 or CS/IL-12 resulted in decreasing IFN- $\gamma$ levels after 2 and 3 injections (Fig. 3B). In contrast, IFN- $\gamma$ levels were not affected by weekly IL-12 or CS/IL-12 dosing via the intravesical route (Fig. 3A). These data suggest that the low systemic IL-12 exposure following intravesical CS/IL-12 immunotherapy does not cause a full release of IFN- $\gamma$ that would result in a refractory period. These data also suggest that weekly CS/IL-12 treatments would be better tolerated and equipotent when administered intravesically.

Dose escalation studies indicated that 5- and 10fold increases in the dose of CS/IL-12, resulted in only minor, statistically insignificant increases in serum IFN- $\gamma$ levels (Fig. 4A). This effect, or lack thereof, was independent of route as neither intravesical nor s.c. CS/IL-12 showed a significant dose effect. These dose-escalation data suggest a potentially wide therapeutic window for intravesical CS/IL-12 immunotherapy.

Even without dose-limiting IL-12 exposure or evidence of systemic toxicity, local inflammatory responses could still affect the utility of CS/IL-12. In fact, 27-95\% of NMIBC patients experience treatment-limiting cystitis following intravesical BCG [36]. Fortunately, our histopathology studies revealed no treatment-related inflammation greater than grade 1 (Table 1). Furthermore, there were no differences in inflammation between any of the treatment groups. These data do not rule out the potential for cystitis in clinical studies, however, the absence of cystitis at this point is at least promising.

The only clinical study to date using intravesical IL-12 was published by Weiss et al. in 2003 [37]. Patients received up to $200 \mu \mathrm{g}$ IL-12 intravesically. If one assumes an average human blood volume of $5 \mathrm{~L}$ and uses the same 540-fold reduction that we found for unformulated IL-12, the theoretical peak concentration of IL-12 would be about $0.07 \mathrm{ng} / \mathrm{ml}$. It is therefore not surprising that IL-12 and IFN- $\gamma$ were not detected in the aforementioned clinical study or in preclinical studies with cynomolgus monkeys [37].

Taken together, results of the above studies support the safety and tolerability of intravesical CS/IL-12 immunotherapy. In addition to its robust antitumor activity $[16,17]$, intravesical CS/IL-12 is well tolerated with a predictable pharmacokinetic profile. These data will be useful in planning future studies in pet dogs with spontaneous invasive urothelial carcinoma of the bladder and in patients with BCGrefractory bladder cancer.

\section{ACKNOWLEDGMENTS}

The authors have no acknowledgments.

\section{FUNDING}

This work was supported by funding from the National Cancer Institute (R01CA172631) and the National Science Foundation Graduate Research Fellowship Program.

This project was funded in part by a grant from the University of North Carolina Lineberger Comprehensive Cancer Center.

\section{AUTHOR CONTRIBUTIONS}

K.G.N., E.S.W., D.M.M., and D.A.Z.: conceptualization, data curation, formal analysis, manuscript preparation.

K.G.N., E.S.W., M.R.V., S.M.M., and D.A.Z.: investigation, methodology.

D.A.Z.: resources, supervision, funding acquisition.

\section{CONFLICT OF INTERESTS}

K.G.N., E.S.W., M.R.V., S.M.M., D.M.M., and D.A.Z. declare that they have no conflicts of interest with the contents of this article.

\section{REFERENCES}

[1] Alexandroff AB, Jackson AM, O'Donnell MA, James K. BCG immunotherapy of bladder cancer: 20 years on. Lancet. 1999;353(9165):1689-94. doi: 10.1016/S01406736(98)07422-4.

[2] Morales A, Eidinger D, Bruce AW. Intracavitary Bacillus Calmette-Guerin in the treatment of superficial bladder tumors. J Urol. 1976;116(2):180-3. doi: 10.1016/s00225347(17)58737-6.

[3] Bohle A, Brandau S. Immune mechanisms in bacillus Calmette-Guerin immunotherapy for superficial bladder cancer. J Urol. 2003;170(3):964-9. doi: 10.1097/01. ju.0000073852.24341.4a.

[4] Bohle A, Gerdes J, Ulmer AJ, Hofstetter AG, Flad HD. Effects of local bacillus Calmette-Guerin therapy in patients with bladder carcinoma on immunocompetent cells of the bladder wall. J Urol. 1990;144(1):53-8. doi: 10.1016/s00225347(17)39365-5.

[5] de Boer EC, de Jong WH, van der Meijden AP, Steerenberg PA, Witjes F, Vegt PD, et al. Leukocytes in the urine after intravesical BCG treatment for superficial bladder cancer. A 
flow cytofluorometric analysis. Urol Res. 1991;19(1):45-50. doi: 10.1007/BF00294021.

[6] Prescott S, James K, Hargreave TB, Chisholm GD, Smyth JF. Intravesical Evans strain BCG therapy: quantitative immunohistochemical analysis of the immune response within the bladder wall. J Urol. 1992;147(6):1636-42. doi: 10.1016/s0022-5347(17)37668-1.

[7] Lamm DL. Long-term results of intravesical therapy for superficial bladder cancer. Urol Clin North Am. 1992; 19(3):573-80.

[8] Malmstrom PU, Wijkstrom H, Lundholm C, Wester K, Busch C, Norlen BJ. 5-year followup of a randomized prospective study comparing mitomycin $\mathrm{C}$ and bacillus Calmette-Guerin in patients with superficial bladder carcinoma. Swedish-Norwegian Bladder Cancer Study Group. J Urol. 1999;161(4):1124-7.

[9] Lasek W, Zagozdzon R, Jakobisiak M. Interleukin 12: still a promising candidate for tumor immunotherapy? Cancer Immunol Immunother. 2014;63(5):419-35. doi: 10.1007/s00262-014-1523-1.

[10] Tugues S, Burkhard SH, Ohs I, Vrohlings M, Nussbaum $\mathrm{K}$, Vom Berg J, et al. New insights into IL-12-mediated tumor suppression. Cell Death Differ. 2015;22(2):237-46. doi: $10.1038 /$ cdd.2014.134.

[11] Nguyen KG, Vrabel MR, Mantooth SM, Hopkins JJ, Wagner ES, Gabaldon TA, et al. Localized Interleukin-12 for Cancer Immunotherapy. Front Immunol. 2020;11:575597. doi: 10.3389/fimmu.2020.575597.

[12] Leonard JP, Sherman ML, Fisher GL, Buchanan LJ, Larsen $\mathrm{G}$, Atkins MB, et al. Effects of single-dose interleukin12 exposure on interleukin-12-associated toxicity and interferon-gamma production. Blood. 1997;90(7):2541-8.

[13] Douglass L, Schoenberg M. The Future of Intravesical Drug Delivery for Non-Muscle Invasive Bladder Cancer. Bladder Cancer. 2016;2(3):285-92. doi: 10.3233/BLC-160056.

[14] Pan Y, Chang T, Marcq G, Liu C, Kiss B, Rouse R, et al. In vivo biodistribution and toxicity of intravesical administration of quantum dots for optical molecular imaging of bladder cancer. Sci Rep. 2017;7(1):9309. doi: 10.1038/ s41598-017-08591-w.

[15] Zaharoff DA, Hoffman BS, Hooper HB, Benjamin CJ, Jr., Khurana KK, Hance KW, et al. Intravesical immunotherapy of superficial bladder cancer with chitosan/interleukin-12. Cancer Res. 2009;69(15):6192-9. doi: 10.1158/0008-5472. CAN-09-1114.

[16] Smith SG, Koppolu BP, Ravindranathan S, Kurtz SL, Yang $\mathrm{L}$, Katz MD, et al. Intravesical chitosan/interleukin-12 immunotherapy induces tumor-specific systemic immunity against murine bladder cancer. Cancer Immunol Immunother. 2015;64(6):689-96. doi: 10.1007/s00262-015-1672-x.

[17] Smith SG, Baltz JL, Koppolu BP, Ravindranathan S, Nguyen K, Zaharoff DA. Immunological mechanisms of intravesical chitosan/interleukin-12 immunotherapy against murine bladder cancer. Oncoimmunology. 2017;6(1):e12 59050. doi: 10.1080/2162402X.2016.1259050.

[18] Valachova K, Soltes L. Versatile Use of Chitosan and Hyaluronan in Medicine. Molecules. 2021;26(4). doi: 10.3390/molecules26041195.

[19] de Sousa Victor R, Marcelo da Cunha Santos A, Viana de Sousa B, de Araujo Neves G, Navarro de Lima Santana L, Rodrigues Menezes R. A Review on Chitosan's Uses as Biomaterial: Tissue Engineering, Drug Delivery Systems and Cancer Treatment. Materials (Basel). 2020;13(21). doi: 10.3390/ma13214995.
[20] Zhang J, Jiang $\mathrm{H}$, Zhang $\mathrm{H}$. In situ administration of cytokine combinations induces tumor regression in mice. EBioMedicine. 2018;37:38-46. doi: 10.1016/j.ebiom.2018. 09.050 .

[21] Alatrash G, Hutson TE, Molto L, Richmond A, Nemec $\mathrm{C}$, Mekhail T, et al. Clinical and immunologic effects of subcutaneously administered interleukin-12 and interferon alfa-2b: phase I trial of patients with metastatic renal cell carcinoma or malignant melanoma. J Clin Oncol. 2004;22(14): 2891-900. doi: 10.1200/JCO.2004.10.045.

[22] Jayanthi S, Koppolu B, Smith SG, Jalah R, Bear J, Rosati M, et al. Efficient production and purification of recombinant human interleukin-12 (IL-12) overexpressed in mammalian cells without affinity tag. Protein Expr Purif. 2014;102:7684. doi: 10.1016/j.pep.2014.07.002.

[23] Jayanthi S, Koppolu BP, Nguyen KG, Smith SG, Felber BK, Kumar TKS, et al. Modulation of Interleukin-12 activity in the presence of heparin. Sci Rep. 2017;7(1): 5360. doi: 10.1038/s41598-017-05382-1.

[24] Nguyen KG, Gillam FB, Hopkins JJ, Jayanthi S, Gundampati RK, Su G, et al. Molecular mechanisms of heparin-induced modulation of human interleukin 12 bioactivity. J Biol Chem. 2019;294(12):4412-24. doi: 10.1074/jbc.RA118.006193.

[25] Schafer KA, Eighmy J, Fikes JD, Halpern WG, Hukkanen RR, Long GG, et al. Use of Severity Grades to Characterize Histopathologic Changes. Toxicol Pathol. 2018;46(3):25665. doi: 10.1177/0192623318761348.

[26] Weiss JM, Subleski JJ, Wigginton JM, Wiltrout RH. Immunotherapy of cancer by IL-12-based cytokine combinations. Expert Opin Biol Ther. 2007;7(11):1705-21. doi: 10.1517/14712598.7.11.1705.

[27] Carson WE, Dierksheide JE, Jabbour S, Anghelina M, Bouchard P, Ku G, et al. Coadministration of interleukin-18 and interleukin-12 induces a fatal inflammatory response in mice: critical role of natural killer cell interferon-gamma production and STAT-mediated signal transduction. Blood. 2000;96(4):1465-73.

[28] Nakamura S, Otani T, Ijiri Y, Motoda R, Kurimoto M, Orita $\mathrm{K}$. IFN-gamma-dependent and -independent mechanisms in adverse effects caused by concomitant administration of IL-18 and IL-12. J Immunol. 2000;164(6):3330-6. doi: 10.4049/jimmunol.164.6.3330.

[29] Hong F, Jaruga B, Kim WH, Radaeva S, El-Assal ON, Tian Z, et al. Opposing roles of STAT1 and STAT3 in T cell-mediated hepatitis: regulation by SOCS. J Clin Invest. 2002;110(10):1503-13. doi: 10.1172/JCI15841.

[30] Jaruga B, Hong F, Sun R, Radaeva S, Gao B. Crucial role of IL-4/STAT6 in T cell-mediated hepatitis: up-regulating eotaxins and IL-5 and recruiting leukocytes. J Immunol. 2003;171(6):3233-44. doi: 10.4049/jimmunol.171.6. 3233.

[31] Portielje JE, Lamers CH, Kruit WH, Sparreboom A, Bolhuis $\mathrm{RL}$, Stoter G, et al. Repeated administrations of interleukin (IL)-12 are associated with persistently elevated plasma levels of IL-10 and declining IFN-gamma, tumor necrosis factor-alpha, IL-6, and IL-8 responses. Clin Cancer Res. 2003;9(1):76-83.

[32] Shi G, Edelblute C, Arpag S, Lundberg C, Heller R. IL12 Gene Electrotransfer Triggers a Change in Immune Response within Mouse Tumors. Cancers (Basel). 2018; 10(12). doi: 10.3390/cancers 10120498.

[33] Little RF, Pluda JM, Wyvill KM, Rodriguez-Chavez IR, Tosato G, Catanzaro AT, et al. Activity of subcutaneous interleukin-12 in AIDS-related Kaposi sarcoma. 
Blood. 2006;107(12):4650-7. doi: 10.1182/blood-2005-114455.

[34] Flecknell PA. Clinical, Biochemical and Haematological Reference Values in Normal Experimental Animals. J Clin Pathol. 1979;32(1):96-.

[35] Dholakia U, Clark-Price SC, Keating SCJ, Stern AW. Anesthetic effects and body weight changes associated with ketamine-xylazine-lidocaine administered to CD-1 mice. PLoS One. 2017;12(9):e0184911. doi: 10.1371/journal.pone.0184911.
[36] Liu Y, Lu J, Huang Y, Ma L. Clinical Spectrum of Complications Induced by Intravesical Immunotherapy of Bacillus Calmette-Guerin for Bladder Cancer. J Oncol. 2019;2019: 6230409. doi: 10.1155/2019/6230409.

[37] Weiss GR, O’Donnell MA, Loughlin K, Zonno K, Laliberte RJ, Sherman ML. Phase 1 study of the intravesical administration of recombinant human interleukin-12 in patients with recurrent superficial transitional cell carcinoma of the bladder. J Immunother. 2003;26(4):343-8. 